\title{
An Anomalous Component of Galactic Emission
}

\author{
E.M. Leitch, A.C.S. Readhead, T.J. Pearson, S.T. Myers ${ }^{1}$ \\ California Institute of Technology, 105-24 \\ Pasadena CA 91125
}

\begin{abstract}
We present results from microwave background observations at the Owens Valley Radio Observatory. These observations, at 14.5 and $32 \mathrm{GHz}$, are designed to detect intrinsic anisotropy on scales of $7^{\prime}-22^{\prime}$. After point source removal, we detect significant emission with temperature spectral index $\beta \simeq-2$ towards the North Celestial Pole (NCP). Comparison of our data with the IRAS $100 \mu \mathrm{m}$ map of the same fields reveals a strong correlation between this emission and the infrared dust emission. From the lack of detectable $\mathrm{H} \alpha$ emission, we conclude that the signals are consistent either with flatspectrum synchrotron radiation, or with free-free emission from $T_{e} \gtrsim 10^{6} \mathrm{~K}$ gas, probably associated with a large HI feature known as the NCP Loop. Assuming $\beta=-2.2$, our data indicate a conversion $T_{f} / I_{100 \mu \mathrm{m}}=7.5 \times 10^{-2} \nu_{\mathrm{GHz}}^{-2.2} \mathrm{~K} /(\mathrm{MJy} / \mathrm{sr})$.

The detection of such a component suggests that we should be cautious in any assumptions made regarding foregrounds when designing experiments to map the microwave background radiation.
\end{abstract}

Subject headings: Dust, extinction - HII regions — supernova remnants - cosmic microwave background

${ }^{1}$ University of Pennsylvania 


\section{Observations}

Since 1993, the Owens Valley Radio Observatory (OVRO) 5.5-m telescope (see Herbig et al. 1995) has been used for extensive observations at $32 \mathrm{GHz}$ of the cosmic microwave background (CMB) on angular scales of $7^{\prime}-22^{\prime}$. The receiver input is switched at $500 \mathrm{~Hz}$ between two beams of $7^{\prime} .35$ (FWHM) separated by $22^{\prime} .16$. The OVRO $40-\mathrm{m}$ telescope, underilluminated at $14.5 \mathrm{GHz}$ to match the $5.5-\mathrm{m}$ beam, provides a second frequency channel for spectral discrimination of foregrounds (see Table 1). Both receivers detect right-circular polarization.

TABLE 1

Parameters for the OVRO 5.5-M AND 40-M telescopes

\begin{tabular}{lll}
\hline \hline & $5.5-\mathrm{m}$ & $40-\mathrm{m}$ \\
\hline $\begin{array}{l}\text { Frequency } \\
\text { Bandwidth }\end{array}$ & $32 \mathrm{GHz}$ & $14.5 \mathrm{GHz}$ \\
$\begin{array}{l}\text { System } \\
\text { temperature }\end{array}$ & $5.6 \mathrm{GHz}$ & $3 \mathrm{GHz}$ \\
$\begin{array}{l}\text { Beam efficiency } \\
\text { Beamwidth }\end{array}$ & 0.61 & $48 \mathrm{~K}$ \\
$\begin{array}{l}\text { FWHM) } \\
\text { Beamthrow }\end{array}$ & $7^{\prime} .35$ & 0.70 \\
$\begin{array}{l}\text { Main beam } \\
\text { solid angle }\end{array}$ & $22^{\prime} .16$ & $7^{\prime} .35$ \\
\hline
\end{tabular}

In the "RING5M" experiment, we observe 36 fields at $\delta \simeq 88^{\circ}$ spaced by the $22^{\prime} .16$ beamthrow in a ring around the NCP (see Figure 1). To minimize variations in differential contributions from the ground, each field is observed only within \pm 20 minutes of upper culmination. In each flux measurement, the telescope moves in azimuth to alternate the beams on the main field; each measurement is thus the difference between the signal from the main field and the average of the signals in the two adjacent fields (Readhead et al. 1989). As a result, a strong signal in one field produces a negative signal half as strong in each of the two flanking fields (see Figure 2).

To estimate the contribution of point sources, the RING5M fields were mapped on the VLA at $8 \mathrm{GHz}$ to a sensitivity of $0.25 \mathrm{mJy}$; a total of 34 sources were detected with $S_{8 \mathrm{GHz}} \gtrsim 2 \mathrm{mJy}$. Subsequent monthly VLA monitoring of these sources at 8 and $15 \mathrm{GHz}$ provided accurate measurements of flux densities and spectral indices, enabling us to estimate the flux densities at both $14.5 \mathrm{GHz}$ and $32 \mathrm{GHz}$ and to subtract the point-source contribution from these data sets.

We report here only on results relevant to an anomalous foreground we have detected. The implications for CMB observations and a full discussion and analysis of our results will be presented in subsequent papers.

\section{Analysis}

Our cumulative observations of the RING5M fields have achieved a $1 \sigma \mathrm{rms}$ sensitivity of $17 \mu \mathrm{K}$ per field at $32 \mathrm{GHz}$, and $15 \mu \mathrm{K}$ per field at $14.5 \mathrm{GHz}$. Signals $\geq 200 \mu \mathrm{K}$ are seen at both frequencies, and excellent reproducibility of these data between the 1994, 1995 and 1996 observing seasons indicates that they represent real structure on the sky.

In addition to good agreement between independent data sets at the same frequency, a Spearman rank test (Kendall 1955), modified to account for correlations introduced by the switching (see $\S 3$ ), finds a correlation $r_{s}=0.84$ between the two frequencies, with a significance $P\left(r_{s} \geq 0.84\right)=7 \times 10^{-7}$. The strength of the observed correlation between independent observations on separate telescopes is further evidence that the signals are astronomical in origin, and not artifacts of the observing procedure.

Since the only common element between the two channels is our observing strategy, we explored the possibility of systematic contamination by observing the RING5M fields at $14.5 \mathrm{GHz}$ for two weeks at lower culmination. The data obtained showed the same signals, to within the observed $\sim 80 \mu \mathrm{K}$ scatter between two-week subsets of our upper culmination data.

In all of the following discussion, we use data sets from which the point-source contributions have been subtracted. Apart from one source, which affected 3 of our 36 fields, the contributions of point sources were much smaller than the detected signals. The maximum $1 \sigma$ error on the estimated point-source contribution to a field was $22 \mu \mathrm{K}$. There is therefore no doubt that we have detected a significant astronomical signal which is not due to point sources.

\subsection{Spectral Index of the Foreground}

The strongest signals seen in both the 14.5 and $32 \mathrm{GHz}$ channels have steep spectral indices and amplitudes $T \sim 300 \mu \mathrm{K}$ at $14.5 \mathrm{GHz}$. A likelihood analysis yields $\beta=-1.1_{-0.3}^{+0.2}$ for the spectral index of the data set as a whole, indicating the presence of significant emission with $\beta<0$.

Recent Westerbork observations (Wieringa et al. 1993) reveal polarized structures at high Galactic lat- 
itude as bright as $8 \mathrm{~K}$ at $325 \mathrm{MHz}$. These features, on scales of $4^{\prime}-30^{\prime}$, are seen in linear polarization only; the corresponding total intensity maps are extremely smooth, and upper limits $<0.5-1 \mathrm{~K}$ are set on any conterparts in total intensity. This is interesting, as $\sim 8 \mathrm{~K}$ features with spectral index $\beta=-2.7$ can just reproduce the observed structures at $14.5 \mathrm{GHz}$ if we were $100 \%$ sensitive to linear polarization. Tests of our $14.5 \mathrm{GHz}$ polarizers, however, indicate $<6 \%$ contamination from linear polarization across our bandpass, so it is clear that such polarized features cannot account for the signals we have detected.

Moreover, given the smoothness of the total intensity maps, it is highly improbable that the structure in the polarized emission is due to variations in intrinsic polarization angle, and the polarized structure is interpreted as Faraday rotation of an intrinsically smooth, polarized synchrotron background by an intervening screen. As a result, the polarization angle will have the $\nu^{-2}$ dependence of Faraday rotation and the structure should be negligible at $14.5 \mathrm{GHz}$.

Total intensity maps from the WENSS survey (de Bruyn 1996), covering 20 of the 36 RING5M fields, show no detectable signals after removal of discrete sources. Comparison of the maximum signal at $14.5 \mathrm{GHz}$ with the rms from the WENSS maps in the overlap fields places a lower limit $\beta \geq-2.2$ on the spectral index of the foreground we have detected.

Thus, based on the WENSS maps, we know that the contribution of any steep-spectrum $(\beta<-2.2)$ component is negligible and we now investigate what foreground spectral index is favored by our data. We model our data as a Gaussian CMB component in the presence of a single foreground of variable strength but constant spectral index $\beta$. Defining $\Delta T_{i}=\delta T_{i}-$ $\frac{1}{2}\left(\delta T_{i+1}+\delta T_{i-1}\right)$, as described in $\S 1$, for each field $i$, we measure

$$
\Delta T_{i}(\nu)=\Delta T_{i_{\text {cmb }}}+\Delta T_{i_{\text {fore }}} \nu^{\beta} .
$$

Given $\Delta T_{i}(\nu)$ measured at two frequencies $\nu_{1}$ and $\nu_{2}$, we can solve for the CMB component in terms of the unknown spectral index of the foreground:

$$
\Delta T_{i_{c m b}}(\beta)=\frac{\Delta T_{i}\left(\nu_{1}\right) \nu_{1}^{-\beta}-\Delta T_{i}\left(\nu_{2}\right) \nu_{2}^{-\beta}}{\nu_{1}^{-\beta}-\nu_{2}^{-\beta}} .
$$

The likelihood function for the CMB component (see,

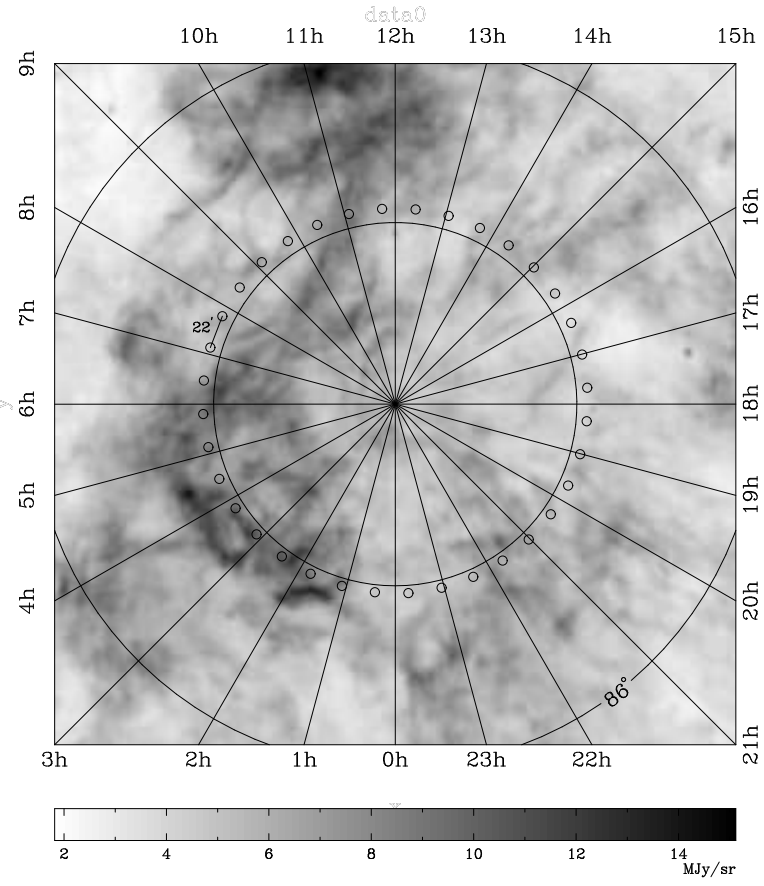

Fig. 1.- IRAS $100 \mu \mathrm{m}$ map in J2000 coordinates, with RING5M fields over-plotted. The spacing of the fields is $\sim 22^{\prime}$, and the FWHM of the beam is $7^{\prime} .35$.

for example, Readhead et al. 1989) is then given by

$\mathrm{E}\left(\sigma_{c m b}, \beta\right)=\prod_{i=1}^{36} \frac{1}{\sqrt{2 \pi\left(\epsilon_{i}^{2}+\sigma_{c m b}^{2}\right)}} \exp \left[-\frac{\Delta T_{i_{c m b}}^{2}(\beta)}{2\left(\epsilon_{i}^{2}+\sigma_{c m b}^{2}\right)}\right]$,

where $\epsilon_{i}$ is the error in the residual CMB component, and $\sigma_{c m b}^{2}$ is the intrinsic CMB variance. The likelihood constructed from the point-source subtracted data sets at 14.5 and $32 \mathrm{GHz}$ peaks at $\beta=-2.25$, with $\beta>-1.8$ ruled out at the $1 \sigma$ level. Our data is thus consistent with a foreground of spectral in$\operatorname{dex} \beta \sim-2$, and we conclude that the foreground is either unusually flat-spectrum synchrotron radiation, or free-free emission.

\section{IRAS $100 \mu \mathrm{m}$ maps}

In an attempt to correlate this component with known Galactic foregrounds, we convolved the IRAS $100 \mu \mathrm{m}$ map (IRAS) of the NCP with our beam and beam-switch. The source-subtracted $14.5 \mathrm{GHz}$ data, along with results of the IRAS convolution, are shown in Figure 2.

We find a clear correlation between the IRAS $100 \mu \mathrm{m}$ maps and our 14.5 GHz data set. To assess 


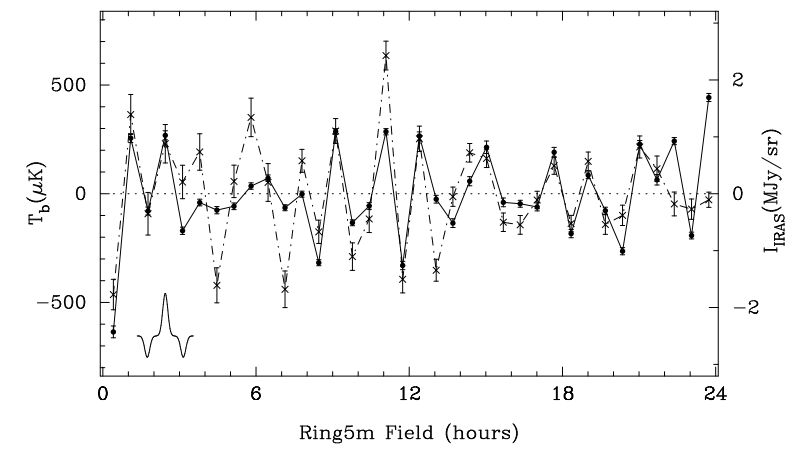

Fig. 2.- Comparison of the $14.5 \mathrm{GHz}$ data (solid line) in $\mu \mathrm{K}$, with the IRAS $100 \mu \mathrm{m}$ convolution (dot-dashed line). Errors for the IRAS data points are the estimated standard deviation of the convolution. The dotted line essentially coincident with the $\mathrm{x}$-axis is the anisotropy inferred from $\mathrm{H} \alpha$ images of the NCP fields, in $\mu \mathrm{K}$. At bottom left is the "triple beam" pattern due to the double switching.

the significance of this result without a priori knowledge of the distribution of the IRAS $100 \mu \mathrm{m}$ brightness or $14.5 \mathrm{GHz}$ temperature on $7^{\prime}$ scales, we use Spearman's rank correlation coefficient $r_{s}$. Since this depends only on the data ranks, whose distribution is known, and not on the values themselves, we can determine the significance of an observed value of $r_{s}$ unambiguously. The observed correlation between the $14.5 \mathrm{GHz}$ and IRAS $100 \mu \mathrm{m}$ data is $r_{s}=0.73$, and, for 36 independent fields, the probability of observing $r_{s} \geq 0.73$ by chance is $P\left(r_{s} \geq 0.73\right)=4.5 \times 10^{-7}$. Due to our switching strategy, however, only every third field is actually independent, and numerical simulations show that this reduces the significance to $6.7 \times 10^{-5}$.

We note that region spanning $3 \mathrm{~h}-8 \mathrm{~h}$, where the correlation is weakest, is also the region where we see the strongest signals at $32 \mathrm{GHz}$; the spectral indices of these fields are consistent with $\beta=0$, indicating the presence of a significant CMB signal.

Taking $\beta=-2.2$ as the spectral index of the foreground most consistent with both our data and the WENSS maps, we can use the $14.5 \mathrm{GHz}$ and $32 \mathrm{GHz}$ data to solve for the free-free component in the manner of Equation (2). A linear fit to the foreground component yields the conversion

$$
T_{f} / I_{100 \mu \mathrm{m}}=7.5 \times 10^{-2} \nu_{\mathrm{GHz}}^{-2.2} \mathrm{~K} /(\mathrm{MJy} / \mathrm{sr}) .
$$

\section{4. $\mathrm{H} \alpha$ Observations of the NCP}

Gaustad et al. (1995) have recently estimated the free-free contamination of small-scale anisotropy ex- periments through $\mathrm{H} \alpha$ observations of the NCP. For the brightness temperature of optically thin hydrogen, we expect

$$
T_{b}(\mu \mathrm{K})=\frac{5.43}{\nu_{10}^{2} T_{4}^{1 / 2}} g_{f f} E M_{\mathrm{cm}^{-6} \mathrm{pc}},
$$

where

$$
g_{f f}=4.69\left(1+0.176 \ln T_{4}-0.118 \ln \nu_{10}\right)
$$

is the free-free Gaunt factor (Spitzer 1978), the frequency is $10^{10} \nu_{10} \mathrm{GHz}$, the electron temperature $T_{e}=$ $10^{4} T_{4} \mathrm{~K}$, and $E M \equiv \int n_{e}^{2} d l$ is the emission measure. For $T_{e} \leq 2.6 \times 10^{4} \mathrm{~K}$, the $\mathrm{H} \alpha$ surface brightness in rayleighs $\left(1 \mathrm{R}=2.42 \times 10^{-7} \mathrm{ergs} \mathrm{cm}^{-2} \mathrm{~s}^{-1} \mathrm{sr}^{-1}\right.$ at $\left.\mathrm{H} \alpha\right)$ is given by

$$
I_{H \alpha}(\mathrm{R})=0.36 E M_{\mathrm{cm}^{-6} \mathrm{pc}} T_{4}^{-0.9}
$$

(Kulkarni \& Heiles 1987).

Simulating our observing procedure on the maps of Gaustad et al. (1995), we measure $\langle\Delta I\rangle_{r m s} \leq 0.1 \mathrm{R}$ on $7^{\prime}$ scales in $\mathrm{H} \alpha$. If we assume $T_{e}=10^{4} \mathrm{~K}$ for the temperature of the emitting gas, the inferred upper limit on the rms at $14.5 \mathrm{GHz}$ due to free-free emission is $\left\langle\Delta T_{f f}\right\rangle_{r m s} \leq 3.2 \mu \mathrm{K}$, a factor of $\sim 60$ lower than the observed $\left\langle\Delta T_{f f}\right\rangle_{r m s}=203 \mu \mathrm{K}$. Furthermore, the $\mathrm{H} \alpha$ maps are featureless; in the 36 RING fields, no signals are seen with $|\Delta I|>0.2 \mathrm{R}$. For the $\sim 300 \mu \mathrm{K}$ signals we detect, Equations (5)-(7) predict an $\mathrm{H} \alpha$ brightness $|\Delta I| \sim 9 \mathrm{R}$.

If considerable dust lies along the line of sight to the NCP, extinction might account for the low levels of observed $\mathrm{H} \alpha$ emission; estimates from the IRAS $100 \mu \mathrm{m}$ intensities, however, imply $\lesssim 0.6$ magnitudes of visual extinction (Simonetti et al. 1996), so that the upper limits on free-free emission can be increased by $74 \%$ at most.

As $T_{e}$ is increased beyond $2.6 \times 10^{4} \mathrm{~K}$, the allowed orbital space for recombination shrinks, and Equation (7) is no longer valid; for $T_{e}>2.6 \times 10^{4} \mathrm{~K}$, a fit to the $\mathrm{H} \alpha$ recombination coefficient gives $\alpha_{H \alpha} \propto T^{-1.2}$ Ferland 1980). The presence of $300 \mu \mathrm{K}$ free-free emission can therefore be reconciled with the observed $3 \sigma \mathrm{H} \alpha$ limit if the emission is due to gas at $T_{e} \gtrsim 10^{6} \mathrm{~K}$. For these temperatures, free-free brightness temperatures of $300 \mu \mathrm{K}$ at $14.5 \mathrm{GHz}$ require an $E M \gtrsim 131$. The corresponding allowed $n_{e}-l$ parameter space is shown in Figure 3. 


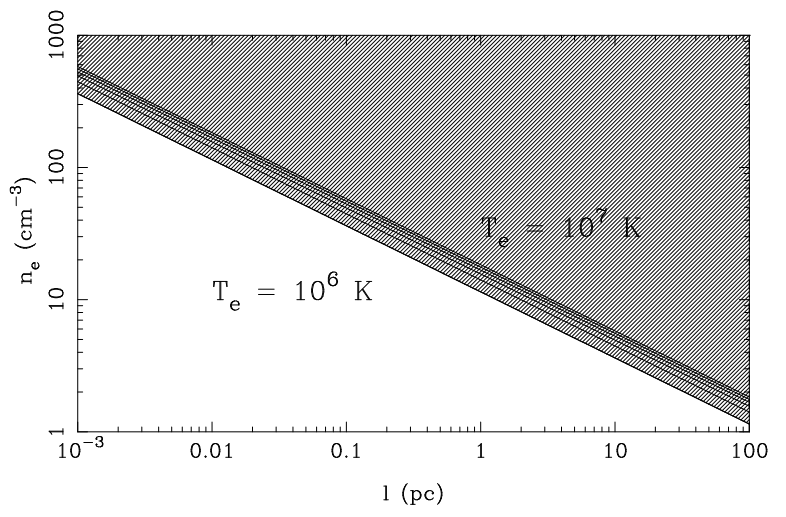

Fig. 3.- Allowed $n_{e}-l$ parameter space (shaded region) for $T_{e} \sim 10^{6}-10^{7} \mathrm{~K}$. We can exclude $l>100 \mathrm{pc}$ as this would require that inhomogeneities be aligned along the line of sight to within $\sim 6 \times 10^{-3} \mathrm{rad}$, since we see fluctuations on the scale of the $22^{\prime}$ beamthrow. The solid lines correspond to $T_{e}=10^{6}, 2 \times 10^{6}, 4 \times 10^{6}, 6 \times 10^{6}, 8 \times 10^{6}$ and $10^{7} \mathrm{~K}$.

\section{Discussion}

The observed structure in the IRAS $100 \mu \mathrm{m}$ map of the NCP region is part of a large HI feature known as the NCP Loop. This feature, which encompasses all of the 36 RING5M fields, has been modeled by Meyerdierks et al. (1991) as the wall of an expanding cylindrical shock. While the production of a dense ionized component such as that implied by Figure 3 may pose significant difficulties — such structures will be extremely overpressured, and must necessarily be transient phenomena - it is intriguing that the combination of large emission measure and high temperature arrived at by interpreting the observed structure at $14.5 \mathrm{GHz}$ as free-free emission are suggestive of just such a shocked component of the ISM.

If the emission is due to $\gtrsim 10^{6} \mathrm{~K}$ gas, this component should have a counterpart in soft X-rays; the absence of any ROSAT PSPC pointings near the NCP and the low resolution of available all-sky surveys, however, prevent any useful comparison with existing data sets.

Perhaps more plausible, though no less anomalous, is the possibility that the observed structure at 14.5 GHz is due to flat-spectrum synchrotron emission. Synchrotron spectral indices as flat as $\beta=-2.0$ are typically observed only in plerions associated with the very youngest SNR (Green 1996), and we would not expect such emission from the NCP Loop - an old remnant, with expansion velocity $v \simeq 20 \mathrm{~km} / \mathrm{s}$. A notable exception to the general steepness of Galac- tic synchrotron radiation, however, is the filamentary structure observed toward the Galactic center. These features, consisting of long, nearly one-dimensional threads, have spectral indices $-2.2 \leq \beta \leq-1.9$ yet show considerable linear polarization, suggesting that the dominant emission mechanism is synchrotron (Yusef-Zadeh 1989). Although such structures would suffer from a similar lifetime problem as free-free filaments and require recent injection of high-energy electrons to maintain a flat spectrum, they would obviate the high temperature and pressure required in the case of free-free emission.

\section{Conclusions}

We have detected a significant correlation between emission with temperature spectral index $\beta \sim-2$ observed at $14.5 \mathrm{GHz}$ in the RING5M experiment, and the IRAS $100 \mu \mathrm{m}$ maps. If this is free-free emission, the lack of accompanying $\mathrm{H} \alpha$ emission implies that it is from a component of the ISM with $T_{e} \gtrsim 10^{6} \mathrm{~K}$. The large EM required to produce the observed signals at these temperatures is typical of SNR, an interpretation supported by the morphology of the NCP Loop, with which the IRAS emission is associated.

Kogut et al. (1996) have recently reported a large angular scale correlation of the residual free-free component in the COBE DMR sky maps with far-infrared DIRBE emission. The level of this signal at $53 \mathrm{GHz}$, however, is consistent with predictions from $\mathrm{H} \alpha$ observations, implying that on $7^{\circ}$ scales, the observed free-free emission is from a $T_{e} \sim 10^{4} \mathrm{~K}$ phase of the ISM. Moreover, if the correlation with free-free emission persists to small scales, the power spectrum of the high-latitude DIRBE $240 \mu \mathrm{m}$ maps $P(\ell) \propto \ell^{-3}$ where $\ell \sim 60 / \theta^{\circ}$, implies a level of free-free at $0.1^{\circ}$ scales marginally consistent with the limit inferred from $\mathrm{H} \alpha$ observations.

If the observed foreground is not unique to the NCP region, our results imply that such emission could be a serious contaminant to small-scale CMB measurements in other areas of sky. This component does, however, have a significantly steeper spectral index and may be subtracted out by multi-frequency observations. Moreover, further observations now in progress to determine the extent of the correlation between $14.5 \mathrm{GHz}$ and $100 \mu \mathrm{m}$ emission indicate that these results for the NCP region are atypical.

We would like to thank A. G. de Bruyn for making 
data from the WENSS survey available to us prior to publication, Gaustad et al. for placing their $\mathrm{H} \alpha$ images in the public domain, C. Heiles for pointing us to the work of Meyerdierks et al, and a referee for a number of useful comments. This research has made use of the SkyView database, operated under the auspices of the High Energy Astrophysics Science Archive Research Center (HEASARC) at the GSFC Laboratory for High Energy Astrophysics. This work at the OVRO is supported by NSF grant number AST 94-19279.

\section{REFERENCES}

de Bruyn, A. G. 1996, IAU Symp. 175 (Extragalactic Radio Sources, ed. R. Ekers et al.), 495

Ferland, G.J. 1980, PASP, 92, 596

Gaustad, J.E., McCullough, P.R., \& Van Buren, D. 1996, PASP, 108, 823

Green, D. A. 1996, A Catalogue of Galactic Supernova Remnants (1996 August version), (Cambridge, UK: Mullard Radio Astronomy Observatory) (available on the World-WideWeb at 'http://www.mrao.cam.ac.uk/surveys/snrs/").

Herbig, T., Lawrence, C. R., Readhead, A. C. S., \& Gulkis, S. 1995, ApJ, 449, L5

IRAS Catalogs and Atlases: Explanatory Supplement (1988, ed. C.A. Beichman, G. Neugebauer, H.J. Habing, P.E. Clegg and T.J. Chester, Washington, DC: GPO)

Kendall, M.G. 1955, Rank Correlation Methods (London: Charles Griffin \& Co. Ltd.)

Kogut, A., et al. 1996, ApJ, 460, 1

Kulkarni, S.R., \& Heiles, C. 1987, in Interstellar Processes, ed. D.J. Hollenbach \& H.A. Thronson, Jr. (Reidel: Dordrecht), 87

Meyerdierks, H., Heithausen, A. \& Reif, K. 1991, A\&A, 245, 247

Press, W.H., Flannery, B.P., Teukolsky, S.A., Vetterling, W.T. 1988, Numerical Recipes in C (Cambridge: Cambridge Univ. Press)

Readhead, A.C.S., Lawrence, C.R., Myers, S.T., Sargent, W.L.W., Hardebeck, H.E. \& Moffet, A.T. 1989, ApJ, 346, 566

Simonetti, J.H., Dennison, B. \& Topansa, G. A. 1996, ApJ, 458, L1

Spitzer, L. 1978, Physical Processes in The Interstellar Medium (New York: John Wiley \& Sons, Inc.)

Wieringa, M.H., de Bruyn, A.G., Jansen, D., Brouw, W.N. \& Katgert, P. 1993, A\&AS, 268, 215

Yusef-Zadeh, F. 1989, in The Center of the Galaxy, ed. M. Morris (Kluwer Academic: Dordrecht), 243
This 2-column preprint was prepared with the AAS IATEX macros v4.0. 